\title{
EFEITO DA ADIÇÃO DE BaO NAS PROPRIEDADES TÉRMICAS E ESTRUTURAIS DO SISTEMA VÍTREO TeO2-Li2O-BaO
}

\section{Anderson Gonçalves ${ }^{1 *}$, Aloisi Somer ${ }^{1}$, João Luiz Gomes Junior ${ }^{1}$, Jaqueline Valeski Gunha ${ }^{1}$, Gerson Kniphoff da Cruz ${ }^{1}$, Andressa Novatski ${ }^{1}$}

1 Universidade Estadual de Ponta Grossa, Ponta Grossa, Paraná, Brasil

*anderg06@gmail.com

Aceito em: 10/12/2017 Publicado em: 15/12/2017

\begin{abstract}
Resumo: Neste estudo avaliamos o efeito do óxido de bário nas propriedades térmicas e estruturais de amostras do sistema vítreo $\mathrm{TeO}_{2}-\mathrm{Li}_{2} \mathrm{O}-\mathrm{BaO}$ obtidas pelo método de melt-quenching e caracterizadas pelas técnicas de Calorimetria Exploratória Diferencial (DSC) e Espectroscopia Raman. Os resultados de Raman mostram que mudanças estruturais de unidades de $\mathrm{TeO}_{4} \mathrm{para} \mathrm{TeO}_{3+1}$ e $\mathrm{TeO}_{3}$ com a criação de oxigênios não ligados (NBO) e perda de conectividade da rede vítrea, ocorrem quando o $\mathrm{BaO}$ substitui o $\mathrm{TeO}_{2}$. Enquanto que, substituindo o $\mathrm{Li}_{2} \mathrm{O}$ por $\mathrm{BaO}$ a conectividade da rede e as unidades de $\mathrm{TeO}_{4}$ são mantidas. Isto influência nas propriedades térmicas dos vidros, onde os resultados de DSC mostram que as maiores variações nos valores da temperatura de transição vítrea $\left(\mathrm{T}_{\mathrm{g}}\right)$ são obtidas para as amostras onde o $\mathrm{BaO}$ substitui o $\mathrm{Li}_{2} \mathrm{O}$, aumentando significativamente os valores de $\mathrm{T}_{\mathrm{g}}$ com o aumento da quantidade de $\mathrm{BaO}$.
\end{abstract}

Keywords Vidros Teluretos; Óxido de bário; DSC; Espectroscopia Raman.

\section{Introdução}

Vidros teluretos têm atraído atenção especial como materiais promissores para aplicações em Fotônica devido suas propriedades como alta estabilidade térmica $\left(\sim 100{ }^{\circ} \mathrm{C}\right)$ [1-3] baixa temperatura de fusão $\left(\sim 700{ }^{\circ} \mathrm{C}\right)$ [4, 5], uma ampla região de transmissão $(\sim 0,35-5 \mu \mathrm{m})[1,6,7]$, baixa energia de fônons quando comparados aos vidros silicatos $\left(\sim 600-850 \mathrm{~cm}^{-1}\right)[1,2,8]$, alto índice de refração linear ( 2) [1, 6, 9, 10] e alta solubilidade para íons terras-raras [11]. Estas propriedades fazem desta família de vidros bons candidatos para dispositivos de chaveamento óptico $[1,2,11]$ e hospedeiro de laser [1, 2, 12].

Tem sido mostrado que a coordenação geométrica dos átomos de Te é fortemente dependente da composição dos vidros e da natureza química do modificador de rede [13, 14]. A adição de um óxido alcalino e um óxido alcalino terroso na rede do vidro óxido causa modificações nas propriedades estruturais, térmicas e ópticas do vidro. Em vidros 
teluretos, essas modificações têm sido associadas com a quebra de ligações Te-O-Te e uma mudança estrutural tridimensional [1417]. A quebra de ligações Te-O-Te promove a transformação de estruturas de $\mathrm{TeO}_{4}$ para $\mathrm{TeO}_{3+1}$ e para $\mathrm{TeO}_{3}$ e aumenta o número de oxigênios não-ligados (NBO) na estrutura vítrea $[15,17]$.

Propriedades estruturais, térmicas e ópticas de vidros teluretos em sistemas binários de $\mathrm{TeO}_{2}-\mathrm{Li}_{2} \mathrm{O}$ e $\mathrm{TeO}_{2}-\mathrm{BaO}$ ou em sistemas ternários onde um terceiro óxido diferente destes é adicionado, são investigadas em muitos trabalhos [2, 13-23]. Com relação as propriedades estruturais, é mostrado que ambos os óxidos causam mudanças na coordenação dos átomos de telúrio, passando de estruturas de $\mathrm{TeO}_{4}$ para $\mathrm{TeO}_{3+1}$ e $\mathrm{TeO}_{3}$ com NBO [16, 17]. Estas mudanças estruturais afetam as demais propriedades de forma diferente para cada óxido. A adição de $\mathrm{Li}_{2} \mathrm{O}$,tanto no sistema binário quanto em sistemas ternários, causa uma redução nos valores da temperatura de transição vítrea $\left(T_{g}\right)[17,23]$, enquanto que a adição de $\mathrm{BaO}$ em sistemas ternários promove um aumento nos valores de $T_{g}[2,13,22]$. Neste estudo investigamos o efeito da adição de $\mathrm{BaO}$ nas propriedades térmicas e estruturais do sistema vítreo $\mathrm{TeO}_{2}-\mathrm{Li}_{2} \mathrm{O}-\mathrm{BaO}$, tanto no caso para a substituição do óxido formador quanto para o caso da substituição do outro óxido modificador.

\section{Materiais e métodos}

As amostras foram separadas em dois grupos e preparadas nas seguintes concentrações: $80 \mathrm{TeO}_{2}-(20-\mathrm{x}) \mathrm{Li}_{2} \mathrm{O}-\mathrm{xBaO}$ (Grupo I) e (80-x) $\mathrm{TeO}_{2}-20 \mathrm{Li}_{2} \mathrm{O}-\mathrm{xBaO}$ (Grupo II), com x = 0, 5, 10, 15 e $20 \mathrm{~mol} \%$ em ambos os grupos. Estas foram obtidas pelo método convencional de meltquenching a partir dos reagentes comerciais em pó $\mathrm{TeO}_{2}$ (Alfa Aesar, +99\%), $\mathrm{Li}_{2} \mathrm{CO}_{3}$ (LAFAN, +99\%) е $\mathrm{BaO}$ (VETEC, +99\%). Os reagentes foram misturados em almofariz de ágata, fundidos a $850{ }^{\circ} \mathrm{C}$ por 30 min usando um cadinho de platina e vertidos em molde de latão previamente aquecido próximo a temperatura de transição vítrea. Cada amostra recebeu tratamento térmico for 4 horas. Os vidros obtidos foram cortados e preparados de acordo com a técnica de análise utilizada. A Tabela 1 apresenta todas as composições nominais das amostras estudadas.

As medidas de Calorimetria Exploratória Diferencial (DSC) foram realizadas utilizando um equipamento da Setaram Instrumentation modelo Labsys Evo, com uma precisão de $4{ }^{\circ} \mathrm{C}$. As medidas foram realizadas sob atmosfera de Argônio com fluxo de 20 mL/min e uma razão de aquecimento de $10{ }^{\circ} \mathrm{C} / \mathrm{min}$, da temperatura ambiente até $600^{\circ} \mathrm{C}$. Foram utilizados cadinhos de alumina e aproximadamente $30 \mathrm{mg}$ de amostra trituradas e separadas com tamanho de partícula $<53 \mu \mathrm{m}$ 
Para as medidas de Espectroscopia Raman foi utilizado um espectrofotômetro da marca Brüker modelo Senterra, com excitação em 532 nm, uma grade de 1200 linhas/mm, microscópio acoplado com uma lente objetiva de 20X com abertura numérica de 0,40. As medidas foram feitas com amostras em bulk, no intervalo de 350 a $900 \mathrm{~cm}^{-1}$ e tempo de integração de 5 s.

\section{Resultados e discussão}

Um parâmetro importante na caracterização de materiais vítreos é a sua temperatura de transição vítrea, a qual foi determinada a partir das curvas de DSC obtidas e que são apresentadas na Figura 1. Os valores determinados são mostrados na Tabela 1.
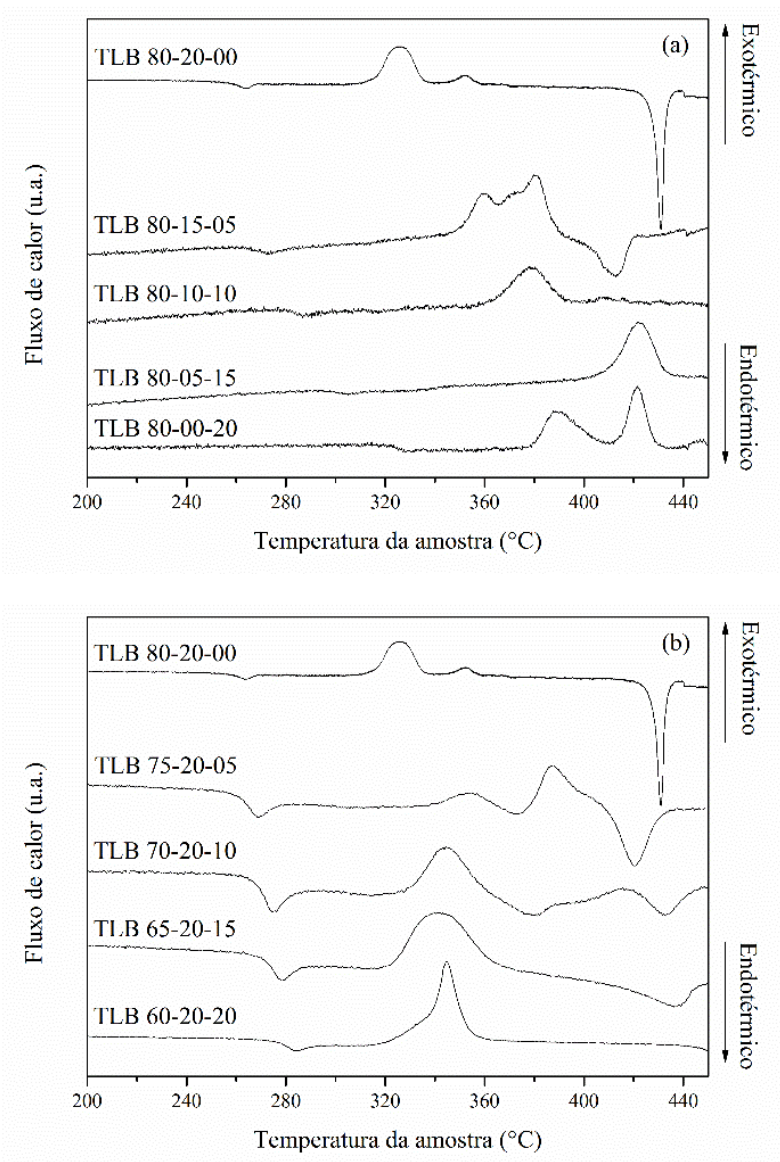

Figura 1 - Curvas de DSC obtidas para as amostras. (a) Grupo I. (b) Grupo II.

Tabela 1 - Composição molar e temperaturas das amostras do sistema ternário $\mathrm{TeO}_{2}-\mathrm{Li}_{2} \mathrm{O}-\mathrm{BaO}$ usadas neste estudo.

\begin{tabular}{|c|c|c|c|c|c|c|}
\hline \multirow{2}{*}{ Nome } & \multicolumn{3}{|c|}{ Composição (mol\%) } & \multirow{2}{*}{$\begin{array}{l}\mathrm{T}_{\mathrm{g}}\left({ }^{\circ} \mathrm{C}\right) \\
( \pm 4)\end{array}$} & \multirow{2}{*}{$\begin{array}{l}T_{x}\left({ }^{\circ} C\right) \\
( \pm 4)\end{array}$} & \multirow{2}{*}{$\begin{array}{l}\Delta \mathrm{T}\left({ }^{\circ} \mathrm{C}\right) \\
( \pm 6)\end{array}$} \\
\hline & $\mathrm{TeO}_{2}$ & $\mathrm{Li}_{2} \mathrm{O}$ & $\mathrm{BaO}$ & & & \\
\hline TLB 80-20-00 & 80 & 20 & 0 & 259 & 316 & 57 \\
\hline TLB 80-15-05 & 80 & 15 & 5 & 264 & 350 & 86 \\
\hline TLB 80-10-10 & 80 & 10 & 10 & 281 & 361 & 80 \\
\hline TLB 80-05-15 & 80 & 5 & 15 & 296 & 409 & 113 \\
\hline TLB 80-00-20 & 80 & 0 & 20 & 321 & 379 & 58 \\
\hline TLB 75-20-05 & 75 & 20 & 5 & 261 & 340 & 79 \\
\hline TLB 70-20-10 & 70 & 20 & 10 & 267 & 330 & 63 \\
\hline TLB 65-20-15 & 65 & 20 & 15 & 270 & 322 & 52 \\
\hline TLB 60-20-20 & 60 & 20 & 20 & 275 & 318 & 43 \\
\hline
\end{tabular}


Para as amostras do Grupo I onde o $\mathrm{BaO}$ substitui o $\mathrm{Li}_{2} \mathrm{O}$, percebe-se um aumento significativo nos valores de $\mathrm{T}_{\mathrm{g}}$ à medida que $\mathrm{a}$ quantidade de $\mathrm{BaO}$ aumenta, sendo esta variação de $62{ }^{\circ} \mathrm{C}$. Para as amostras do Grupo II, onde o $\mathrm{BaO}$ substitui o $\mathrm{TeO}_{2}$, também ocorre um aumento nos valores de $T_{g}$, porém em menor escala (variação de $16{ }^{\circ} \mathrm{C}$ ). Esta diferença de comportamento nos dois grupos é melhor percebida na Figura 2.

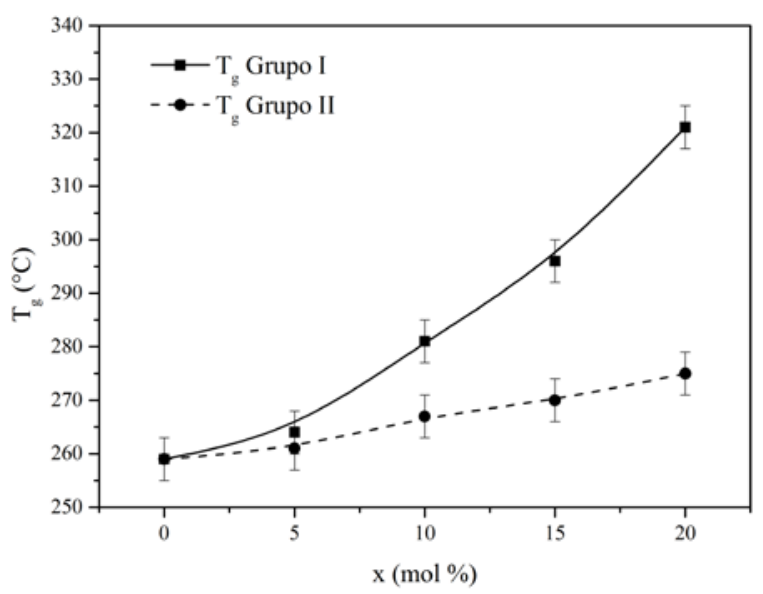

Figura 2 - Variação de $T_{g}$ em função da variação de $\mathrm{x}$.

Este aumento nas temperaturas de $\mathrm{T}_{\mathrm{g}}$ para os dois grupos é devido ao aumento na densidade de ligações cruzadas e forças de ligações entre os átomos envolvidos [2, 24]. A massa molar, relativamente maior, dos átomos de Ba também reduz o volume livre e favorece estas ligações cruzadas, requerendo então mais energia externa para que ocorram mudanças estruturais. Isto leva ao aumento nos valores de $\mathrm{T}_{\mathrm{g}}$ com o aumento da concentração de $\mathrm{BaO}[2$, 22].
A diferença entre os aumentos nos dois casos é devido as quantidades de $\mathrm{Li}_{2} \mathrm{O}$. Ambos os óxidos de bário e lítio causam a ruptura na rede vítrea e consequentemente a criação de $\mathrm{NBO}$, porém as rupturas causadas pelo $\mathrm{Li}_{2} \mathrm{O}$ fazem com que a densidade de ligações cruzadas diminua, resultando em uma perda de conectividade da rede vítrea e diminuição dos valores de $T_{g}[23]$. No Grupo I à medida que o $\mathrm{BaO}$ é adicionado, a quantidade de $\mathrm{Li}_{2} \mathrm{O}$ diminui, assim o efeito do $\mathrm{BaO}$ sobre as temperaturas de $T_{g}$ é maior e provoca a maior variação. No Grupo II, a quantidade de $\mathrm{Li}_{2} \mathrm{O}$ não varia, assim o efeito do $\mathrm{BaO}$ em aumentar os valores de $\mathrm{T}_{\mathrm{g}}$ é reduzido pelo efeito do $\mathrm{Li}_{2} \mathrm{O}$, fazendo com que o aumento não seja tão significativo.

As temperaturas de início de cristalização $\left(\mathrm{T}_{\mathrm{x}}\right)$ também foram determinadas, e seus valores estão na Tabela 1. Para o Grupo I observa-se que esta aumenta os valores com o aumento de $\mathrm{BaO}$ até $15 \%$ deste óxido e depois diminui. Para o Grupo II, ocorre um aumento ao se adicionar $5 \%$ de $\mathrm{BaO}$ e depois a medida que a concentração deste oxido aumenta os valores de $T_{x}$ diminuem. Como descrito anteriormente, no Grupo I a adição de $\mathrm{BaO}$ diminui o volume livre deixando a rede vítrea mais compacta/ densa dificultando o rearranjo molecular [2]. No Grupo II, o efeito do $\mathrm{Li}_{2} \mathrm{O}$ de quebrar ligações e aumentar o volume livre, deixando a rede vítrea mais aberta [23], se torna maior que o efeito do $\mathrm{BaO}$ e faz com que os valores de $\mathrm{T}_{\mathrm{x}}$ diminuam a partir de $5 \%$ de $\mathrm{BaO}$. 
Outro fator que pode influenciar na diminuição destes valores é que neste grupo a quantidade do óxido formador diminui, com isto habilidade de formação vítrea do sistema também diminui [23].

A partir das temperaturas de $T_{g}$ e $T_{x}$ é calculada a estabilidade térmica dos vidros, a qual é definida como $\Delta \mathrm{T}=\mathrm{T}_{\mathrm{x}}-\mathrm{T}_{\mathrm{g}}$ [25], e tem seus valores mostrados na Tabela 1. A estabilidade térmica de um vidro é entendida como a medida da capacidade do vidro de ser processado sem que haja sua cristalização, portanto representa uma faixa de trabalho do vidro. Observa-se da Figura 3 que o comportamento de $\Delta \mathrm{T}$ é maior influenciado pelo comportamento de $\mathrm{T}_{\mathrm{x}}$, uma vez que apresenta comportamento semelhante. No Grupo I ocorre um aumento nos valores até a amostra com $15 \%$ de $\mathrm{BaO}$ e depois uma grande redução. No Grupo II ocorre um aumento para a amostra com $5 \%$ de $\mathrm{BaO}$ e depois os valores diminuem. $\mathrm{O}$ aumento nos valores de $\mathrm{T}_{\mathrm{g}}$ que $\mathrm{o}$ $\mathrm{BaO}$ causa não ocorre da mesma maneira para os valores de $T_{x}$, onde o $\mathrm{Li}_{2} \mathrm{O}$ tem um efeito maior principalmente nas amostras do Grupo II. Isto faz com que os valores de $\Delta \mathrm{T}$ sejam reduzidos para este grupo. Para o Grupo I, onde a influência do $\mathrm{BaO}$ nos valores de $\mathrm{T}_{\mathrm{x}}$ é maior, obtém-se amostras com melhor estabilidade térmica, destacando a amostra TLB 80-05-15 com $\Delta \mathrm{T}$ igual a $113^{\circ} \mathrm{C}$.

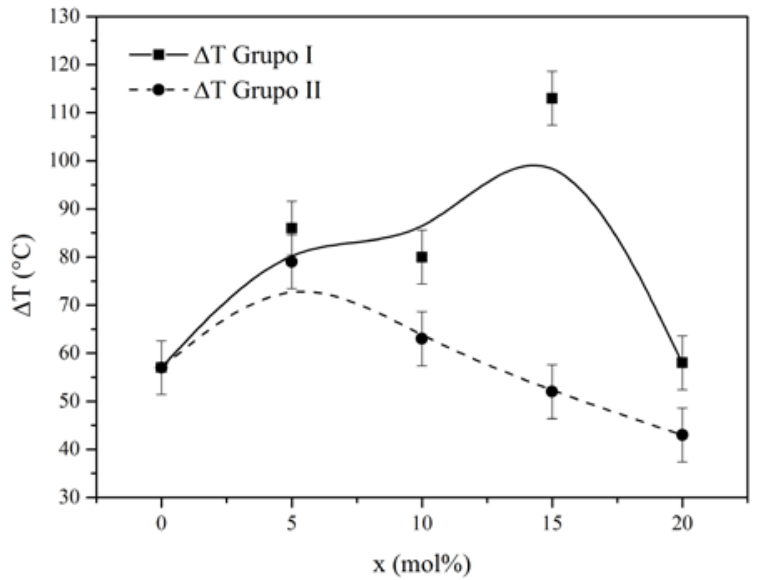

Figura 3 - Variação de $\Delta \mathrm{T}$ em função da variação de $x$.

Os espectros Raman obtidos no intervalo entre 350-900 $\mathrm{cm}^{-1}$ são apresentados nas Figuras 4(a) e 5(a) para as amostras do Grupo I e Grupo II, respectivamente. Para a análise, cada espectro foi normalizado através da razão entre o espectro obtido e a área sob a curva da região analisada. Para explorar os resultados, estes foram deconvoluídos em diferentes bandas gaussianas com base nos dados encontrados na literatura e analisada a evolução de suas áreas em função da adição de BaO. Foram atribuídos cinco bandas às diferentes unidades de $\mathrm{TeO}_{2}[2,7,13,15-17]$, as quais estão centrados em 460, 610, 665, 720 e $775 \mathrm{~cm}^{-1}$. A Figura 4(a) mostra como exemplo a deconvolução feita para a amostra TLB 8020-00. A evolução das áreas dos picos pode ser observada nas Figuras 4(b) e 5(b) para o Grupo I e Grupo II respectivamente. 

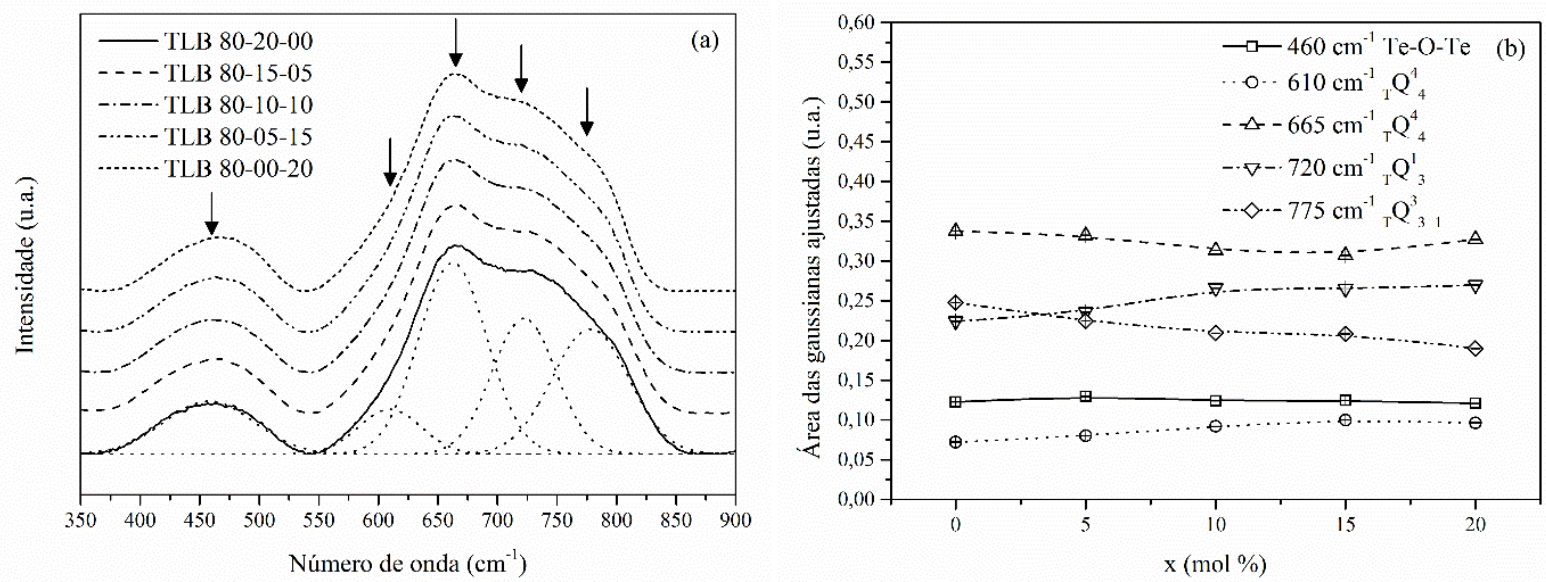

Figura 4 - (a) Espectros Raman obtidos para as amostras do Grupo I. O ajuste gaussiano para as cinco bandas em 460, 610, 665, 720 e $775 \mathrm{~cm}^{-1}$ para a amostra TLB 80-20-00 também é apresentado. As setas indicam as posições destas bandas. (b) Variação nas áreas das bandas em função da variação de $\mathrm{X}$.
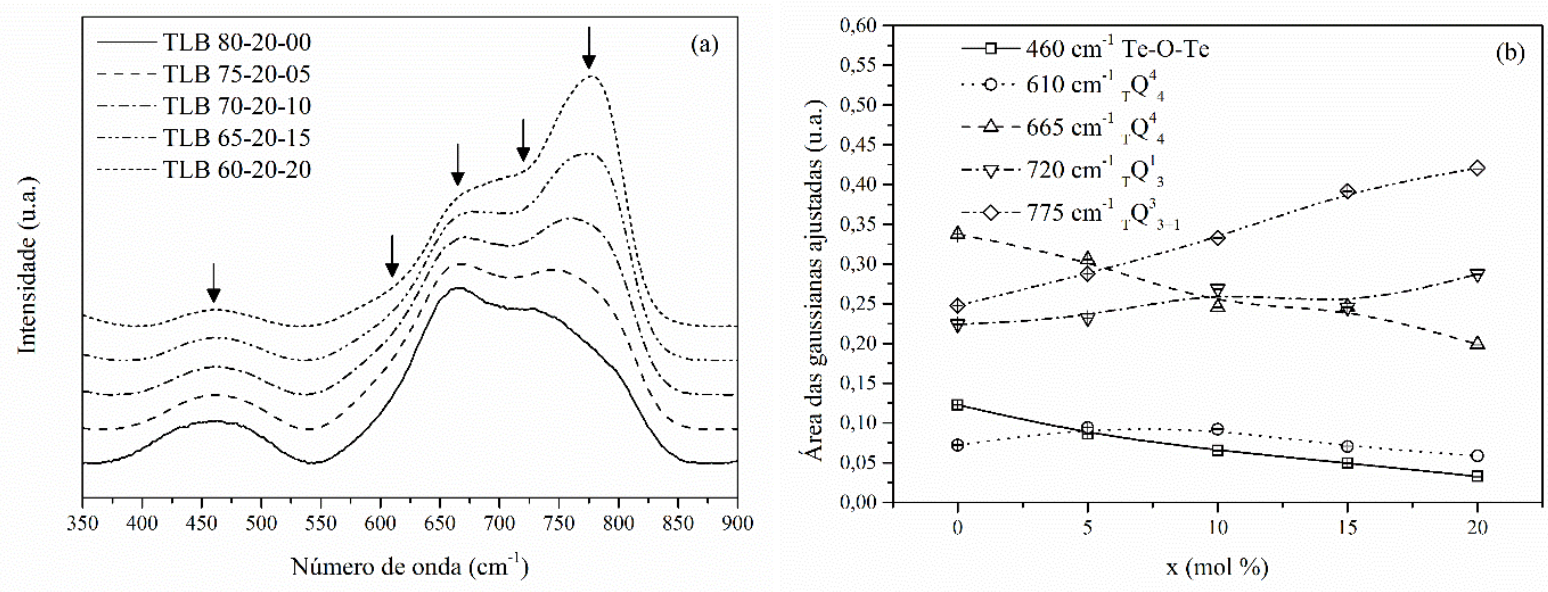

Figura 5 - (a) Espectros Raman obtidos para as amostras do Grupo II. As setas indicam as posições das bandas em 460, 610, 665, 720 e 775 cm-1 . (b) Variação nas áreas das bandas em função da variação de $x$.

Para diferenciar as unidades estruturais na discussão destes resultados será utilizada a notação ${ }_{A} Q_{m}^{n}$, baseada nas referências [15, 26], onde $m$ é o número de coordenação da estrutura, $n$ é o número de oxigênio ligantes e $A$ é o átomo que a unidade se refere, a diferença entre $m$ e $n$ é igual ao número de NBO.

A banda em $460 \mathrm{~cm}^{-1}$ é atribuída aos modos de vibração do tipo flexão simétrica e estiramento de ligações tipo Te-O-Te formadas pelo compartilhamento de vértices de unidades 
estruturais ${ }_{T} Q_{4}^{4},{ }_{T} Q_{3+1}^{3}$ e ${ }_{T} Q_{3}^{1}$ [7]. Assim esta banda é relacionada à conectividade da rede formada. Observa-se que para as amostras do Grupo I não ocorrem grandes variações na área desta banda, isto indica que a conectividade da rede vítrea está sendo mantida a medida que o $\mathrm{BaO}$ substitui o $\mathrm{Li}_{2} \mathrm{O}$. Para as amostras do Grupo II a área desta banda apresenta uma diminuição com o aumento de $\mathrm{BaO}$, diminuindo a conectividade da rede. Esta diminuição observada é atribuída à quebra das ligações Te-O-Te pela ação dos óxidos modificadores que transformam as unidades de ${ }_{T} Q_{4}^{4}$ em unidades de ${ }_{T} Q_{3+1}^{3}$ e ${ }_{T} Q_{3}^{1}$ e criam NBO, os quais diminuem a conectividade da rede $[16,17]$.

Estes resultados reforçam o que foi discutido para os resultados de DSC com relação ao efeito do $\mathrm{BaO}$ sobre os valores de $\mathrm{T}_{\mathrm{g}}$. Para o Grupo I onde não ocorre perda de conectividade, o efeito do $\mathrm{BaO}$ em aumentar estes valores não é afetado, com isso as amostras apresentam maiores variações nos valores de $\mathrm{T}_{\mathrm{g}}$. Para o Grupo II, a perda de conectividade resulta em um decréscimo nos valores de $\mathrm{T}_{\mathrm{g}}$, com a rede menos ligada uma menor energia é necessária para que ocorram mudanças estruturais. Com isto, o aumento nestes valores que ocorre pela adição do $\mathrm{BaO}$ tem o seu efeito reduzido pela perda de conectividade da rede e as amostras apresentam uma menor variação nos valores de $\mathrm{T}_{\mathrm{g}}$ em comparação às do Grupo I.
A banda em $610 \mathrm{~cm}^{-1}$ é atribuída a vibração da rede contínua composta por unidades de ${ }_{T} Q_{4}^{4}[7,16]$, sendo assim associada a conectividade destas unidades. Para o Grupo I observa-se um pequeno aumento na área deste pico até a amostra com $15 \%$ de $\mathrm{BaO}$ e então o valor permanece constante. Este pequeno aumento observado, indica que a adição de $\mathrm{BaO}$ está promovendo um aumento na conectividade destas unidades. No trabalho de Grelowska e colaboradores[13], são estudadas as propriedades estruturais de vidros teluretos no sistema $\mathrm{TeO}_{2}-\mathrm{BaO}-\mathrm{Na}_{2} \mathrm{O}$. Os autores também observam um aumento nos valores da área associada a estas unidades de ${ }_{T} Q_{4}^{4}$ a medida que o $\mathrm{BaO}$ substitui o $\mathrm{Na}_{2} \mathrm{O}$, como os resultados aqui obtidos. Ainda segundo os autores, este aumento indica um aumento no grau de polimerização da rede vítrea formada[13]. Para o Grupo II a área da banda aumenta para a amostra com 5\% de $\mathrm{BaO}$ e depois diminui o seu valor à medida que a quantidade de $\mathrm{BaO}$ aumenta. $\mathrm{O}$ aumento observado na área da banda para a amostra com $5 \%$ de $\mathrm{BaO}$ indica que a quantidade de óxido formador ainda é suficiente para que a polimerização da rede aumente com a adição de $\mathrm{BaO}$, como observado para as amostras do Grupo I. Para as demais amostras do Grupo II a quantidade de $\mathrm{TeO}_{2}$ continua diminuindo e não é suficiente para contrapor o efeito dos óxidos modificadores, com isso a conectividade entre 
as unidades estruturais de ${ }_{T} Q_{4}^{4}$ diminui à medida que o $\mathrm{BaO}$ aumenta.

Outra banda analisada está em 665 cm

1. Esta banda é relacionada às vibrações antissimétricas de ligações $\mathrm{Te}-{ }_{\mathrm{ax}} \mathrm{O}_{\mathrm{eq}}-\mathrm{Te}$, as quais são associadas a presença de unidades de ${ }_{T} Q_{4}^{4}$. Assim esta banda representa a concentração deste tipo de unidades [7]. Para as amostras do Grupo I a área desta banda apresenta apenas pequenas variações com o aumento de $x$. Enquanto que, para o Grupo II os valores apresentam uma diminuição significativa com o aumento da quantidade de $\mathrm{BaO}$, indicando que a quantidade destas unidades estruturais está diminuindo. Esta diminuição também é relacionada à ação dos óxidos modificadores que provocam a mudança de estruturas de ${ }_{T} Q_{4}^{4}$ para estruturas de ${ }_{T} Q_{3+1}^{3}$ e ${ }_{T} Q_{3}^{1}$ pela quebra de ligações do tipo Te-O-Te.

As bandas em 720 e $775 \mathrm{~cm}^{-1}$ são atribuídas a vibrações do tipo estiramento em ligações Te-O- (NBO) presentes em estruturas de ${ }_{T} Q_{3}^{1}$ e ${ }_{T} Q_{3+1}^{3}$, respectivamente [7]. Para o Grupo I, à medida que a quantidade de $\mathrm{BaO}$ aumenta, o valor da área da banda em $720 \mathrm{~cm}^{-1}$ apresenta um aumento até a amostra com 10\% de $\mathrm{BaO}$ e depois permanece constante, enquanto que para a banda em $775 \mathrm{~cm}^{-1}$ é observada uma diminuição nos valores. Para o Grupo II, ambas as bandas apresentam um aumento nos valores de suas áreas à medida que a quantidade de $\mathrm{BaO}$ aumenta, porém, para a banda em $775 \mathrm{~cm}^{-1}$ este aumento é maior.

O aumento na área destas bandas, observado para as amostras do Grupo II, está de acordo com o que foi discutido anteriormente. A medida que os óxidos modificadores são inseridos, ocorre a conversão de unidades de ${ }_{T} Q_{4}^{4} \quad$ para unidades de ${ }_{T} Q_{3+1}^{3} \quad$ e ${ }_{T} Q_{3}^{1}$, consequentemente as áreas das bandas relacionadas à estas estruturas aumentam. Para o Grupo I, o aumento nos valores da área em $720 \mathrm{~cm}^{-1}$ e a diminuição da área em $775 \mathrm{~cm}^{-1}$, ocorrendo ainda uma inversão no comportamento destas, sugere que para as amostras deste grupo, outros processos estão envolvidos na formação das unidades de ${ }_{T} Q_{3+1}^{3}$ e ${ }_{T} Q_{3}^{1}$ à medida que o $\mathrm{BaO}$ substitui o $\mathrm{Li}_{2} \mathrm{O}$.

Estudos anteriores sobre as propriedades estruturais de vidros contendo $\mathrm{TeO}_{2}$ e $\mathrm{BaO}[14$, 27] reportam que para cada átomo de bário na rede vítrea, deve corresponder a dois NBO carregados negativamente $\left({ }_{T} Q_{3+1}^{3}\right)$ e um átomo de telúrio tríplice coordenado $\left({ }_{T} Q_{3}^{1}\right)$. Isto explica o aumento na formação de unidades de ${ }_{T} Q_{3}^{1}$ à medida que a quantidade de $\mathrm{BaO}$ aumenta. Sekiya e colaboradores [16] sugerem que, para vidros com baixa concentração de $\mathrm{BaO}$, podem existir unidades de ${ }_{T} Q_{4}^{4}$ menos distorcidas e contendo uma ligação $\mathrm{Te}_{-\mathrm{eq}} \mathrm{O}^{-}$, 
sendo assim unidades do tipo ${ }_{T} Q_{4}^{3}$. Ainda segundo os autores, estas unidades são formadas pela ruptura de ligações $\mathrm{Te}-{ }_{\mathrm{eq}} \mathrm{O}_{\mathrm{ax}}-\mathrm{Te}$ e proporcionam uma mudança na intensidade da banda em $665 \mathrm{~cm}^{-1}$. A mudança na polarizabilidade é maior para uma ligação do tipo $\mathrm{Te}_{-\mathrm{eq}} \mathrm{O}^{-}$do que para uma ligação $\mathrm{Te}_{\text {eq }} \mathrm{O}$, assim a formação destas unidades de ${ }_{T} Q_{4}^{3}$ pode provocar um aumento na intensidade da banda em $665 \mathrm{~cm}^{-1}$. Portanto sugerimos que para as amostras do Grupo I, à medida que o $\mathrm{BaO}$ substitui o $\mathrm{Li}_{2} \mathrm{O}$, ocorre a diminuição da formação de unidade de ${ }_{T} Q_{3+1}^{3}$ e mais unidades de ${ }_{T} Q_{4}^{3}$ são formadas, diminuindo então os valores da área da banda em $775 \mathrm{~cm}^{-1}$ e provocando a variação nos valores da área da banda em $665 \mathrm{~cm}^{-1}$, como observado.

\section{Conclusão}

Os resultados para as propriedades térmicas estão de acordo com resultados estruturais obtidos. Para o Grupo I, onde a conectividade da rede vítrea é mantida, o efeito do $\mathrm{BaO}$ em aumentar os valores de $T_{g}$ é maior e ocorrem as maiores variações destas temperaturas. Essa conectividade da rede foi confirmada pelos resultados de espectroscopia Raman, nos quais o comportamento da área das bandas relacionados às vibrações de ligações do tipo Te-O-Te e às estruturas de ${ }_{T} Q_{4}^{4}$ não apresentam grandes mudanças, indicando que a

conectividade da rede e a quantidade destas estruturas, em geral, são mantidas.

Já para o Grupo II, onde ocorre uma perda de conectividade que contribui para a diminuição dos valores de $\mathrm{T}_{\mathrm{g}}$, o efeito do $\mathrm{BaO}$ é reduzido e as variações nos valores desta temperatura são menores. Os valores de $\Delta \mathrm{T}$ obtidos para este grupo são menores do que para o Grupo I, onde o $\mathrm{BaO}$ apresenta um maior efeito e obtém-se amostras com melhor estabilidade térmica. Pelos resultados de Raman foi observado um aumento nas áreas das bandas relacionados às unidades estruturais de ${ }_{T} Q_{3+1}^{3}$ e ${ }_{T} Q_{3}^{1}$, isto resulta na formação de NBO que diminuem a conectividade da rede vítrea. A formação destas unidades se dá pela ação dos óxidos modificadores em quebrar as ligações do tipo Te-O-Te formadas por unidades de ${ }_{T} Q_{4}^{4}$, as quais apresentam uma diminuição nos valores das áreas de suas bandas.

\section{Agradecimentos}

Os autores agradecem as agências CNPq, CAPES, FINEP e Fundação Araucária pelo suporte financeiro deste trabalho. Os autores agradecem também ao C-Labmu-UEPG e CRRQ-UEPG pelo suporte técnico.

\section{Referências}


[1] J.S. Wang, E.M. Vogel, E. Snitzer. (1994). Tellurite glass: a new candidate for fiber devices. Optical Materials, 3(3), 187-203.

[2] N. Manikandan, A. Ryasnyanskiy, J. Toulouse. (2012). Thermal and optical properties of $\mathrm{TeO}-\mathrm{ZnO}-\mathrm{BaO}$ glasses. Journal of Non-Crystalline Solids, 358(5), 947-951.

[3] R.A.H. El-Mallawany. (2002). Tellurite Glasses Handbook: Physical Properties and Data. Boca Raton: CRC Press.

[4] N. Boubata, A. Roula, I. Moussaoui. (2013). Thermodynamic and relative approach to compute glass-forming ability of oxides. Bull Mater Sci, 36(3), 457-460.

[5] R. El-Mallawany. (1995). Devitrification and vitrification of tellurite glasses. $J$ Mater Sci: Mater Electron, 6(1), 1-3.

[6] K. Tanaka, K. Kashima, K. Hirao, N. Soga, A. Mito, H. Nasu. (1995). Second harmonic generation in electrically poled Li2O-Nb2O5-TeO2 glasses. Journal of Non-Crystalline Solids, 185(1-2), 123126.

[7] S. Manning, H. Ebendorff-Heidepriem, T.M. Monro. (2012). Ternary tellurite glasses for the fabrication of nonlinear optical fibres. Opt. Mater. Express, 2(2), 140-152.

[8] L.M.S. El-Deen, M.S.A. Salhi, M.M. Elkholy. (2008). IR and UV spectral studies for rare earths-doped tellurite glasses. Journal of Alloys and Compounds, 465(1-2), 333-339.

[9] H. Bürger, K. Kneipp, H. Hobert, W. Vogel, V. Kozhukharov, S. Neov. (1992). Glass formation, properties and structure of glasses in the $\mathrm{TeO}-\mathrm{ZnO}$ system. Journal of Non-Crystalline Solids, 151(1), 134-142.

[10] V. Kozhukharov, M. Marinov, I. Gugov, H. Bürger, W. Vogel. (1983). A new family of tellurife glasses. J Mater Sci, 18(5), 1557-1563.

[11] J.S. Wang, E.M. Vogel, E. Snitzer, J.L. Jackel, V.L. da Silva, Y. Silberberg. (1994). $1.3 \mu \mathrm{m}$ emission of neodymium and praseodymium in tellurite-based glasses. Journal of Non-Crystalline Solids, 178, 109-113.

[12] M. Tatsumisago, S.-K. Lee, T. Minami, Y. Kowada. (1994). Raman spectra of TeO2-based glasses and glassy liquids: local structure change with temperature in relation to fragility of liquid. Journal of Non-Crystalline Solids, 177, 154-163. 
[13] I. Grelowska, M. Reben, B. Burtan, M. Sitarz, J. Cisowski, E.S. Yousef, A. Knapik, M. Dudek. (2016). Structural and optical study of tellurite-barium glasses. Journal of Molecular Structure, 1126, 219-225.

[14] V.O. Sokolov, V.G. Plotnichenko, V.V. Koltashev. (2009). Structure of barium chloride-oxide tellurite glasses. Journal of Non-Crystalline Solids, 355(31-33), 1574-1584.

[15] J.L.J. Gomes, R.L.S. Piazzetta, A. Gonçalves, A. Somer, G.K. da Cruz, F.C. Serbena, A. Novatski. (2015). Correlation between nonbridging oxygens and the thermal and optical properties of the TeO2-Li2O-MoO3 glassy system. Journal of Materials Research, 30(16), 2417-2424.

[16] T. Sekiya, N. Mochida, A. Ohtsuka. (1994). Raman spectra of MO-TeO2 (M = Mg, Sr, Ba and Zn) glasses. Journal of Non-Crystalline Solids, 168(1-2), 106114.

[17] T. Sekiya, N. Mochida, A. Ohtsuka, M. Tonokawa. (1992). Raman spectra of MO1/2TeO2 (M = Li, Na, K, Rb, Cs and Tl) glasses. Journal of Non-Crystalline Solids, 144, 128-144.
[18] Y. Dimitriev, V. Dimitrov, M. Arnaudov. (1983). IR spectra and structures of tellurite glasses. J Mater Sci, 18(5), 1353-1358.

[19] A. Nishara Begum, V. Rajendran. (2007). Structure investigation of TeO2BaO glass employing ultrasonic study. Materials Letters, 61(11-12), 2143-2146.

[20] K. Tanaka, T. Yoko, H. Yamada, K. Kamiya. (1988). Structure and ionic conductivity of $\mathrm{LiCl}-\mathrm{Li} 2 \mathrm{O}-\mathrm{TeO} 2$ glasses. Journal of Non-Crystalline Solids, 103(23), 250-256.

[21] E. Idalgo, E.B. Araújo, K. Yukimitu, J.C.S. Moraes, V.C.S. Reynoso, C.L. Carvalho. (2006). Effects of the particle size and nucleation temperature on tellurite 20Li2O-80TeO2 glass crystallization. Materials Science and Engineering: A, 434(1-2), 13-18.

[22] V. Sreenivasulu, G. Upender, V. Chandra Mouli, M. Prasad. (2015). Structural, thermal and optical properties of $\mathrm{TeO}-\mathrm{ZnO}-\mathrm{CdO}-\mathrm{BaO}$ glasses doped with VO2+. Spectrochimica Acta Part A: Molecular and Biomolecular Spectroscopy, 148, 215222. 
[23] M. Çelikbilek, A.E. Ersundu, S. Aydin. (2013). Preparation and characterization of TeO2-WO3-Li2O glasses. Journal of Non-Crystalline Solids, 378, 247-253.

[24] N.H. Ray. (1974). Compositionproperty relationships in inorganic oxide glasses. Journal of Non-Crystalline Solids, 15(3), 423-434.

[25] A. Dietzel. (1968). Glass structure and glass properties. Glasstech. Ber., 22, 41.

[26] A. Gulenko, O. Masson, A. Berghout, D. Hamani, P. Thomas. (2014). Atomistic simulations of TeO2-based glasses: interatomic potentials and molecular dynamics. Physical Chemistry Chemical Physics, 16(27), 14150-14160.

[27] T. Hayakawa, M. Hayakawa, M. Nogami, P. Thomas. (2010). Nonlinear optical properties and glass structure for MO-Nb2O5-TeO2 (M = Zn, Mg, Ca, Sr, Ba) glasses. Optical Materials, 32(3), 448455. 